\title{
The effects of a nutrition education curriculum on improving young children's fruit and vegetable preferences and nutrition and health knowledge
}

\author{
Sara A Schmitt" ,* Lindsey M Bryant ${ }^{1}$, Irem Korucu ${ }^{1}$, Lisa Kirkham², Bhagyashree Katare ${ }^{3}$ \\ and Tamara Benjamin ${ }^{4}$ \\ 'Human Development and Family Studies, Purdue University, 1202 W. State Street, West Lafayette, IN 47907, \\ USA: ${ }^{2}$ Evaluation and Learning Research Center, College of Education, Purdue University, West Lafayette, IN, USA: \\ ${ }^{3}$ Agricultural Economics, Purdue University, West Lafayette, IN, USA: ${ }^{4}$ Horticulture and Landscape Architecture, \\ West Lafayette, IN, USA
}

Submitted 6 February 2018: Final revision received 15 August 2018: Accepted 3 September 2018: First published online 22 0ctober 2018

\begin{abstract}
Objective: The main objective of the present study was to develop and evaluate a nutrition education curriculum to enhance young children's dietary behaviours and nutrition and health knowledge.

Design: A randomized controlled design was utilized. The intervention was designed to improve children's nutrition and health knowledge and preferences for fruits and vegetables through classroom lessons and activities, including direct instruction and tastings (implemented twice per week for 6 weeks).

Setting: The study took place in elementary schools in a Midwestern US state.

Subjects: One hundred and thirty-one 2 nd grade children from ten classrooms ( $n$ 82 intervention, $n 49$ control) participated.

Results: Multiple regression analyses that adjusted SE for clustering by classroom were used to test hypotheses. Children in the intervention condition demonstrated significantly higher scores on the nutrition and health survey $(\beta=0 \cdot 47, P=0 \cdot 001)$ and showed greater preferences for fruits and vegetables at post-test than the control group $(\beta=0 \cdot 19, P=0 \cdot 003)$.

Conclusions: Findings suggest that a short, 6 -week intervention that aligns with educational standards has the ability to significantly enhance children's outcomes and thus may be a more feasible option for teachers to incorporate into their classrooms than what is currently available.
\end{abstract}

\author{
Keywords \\ Nutrition education \\ Dietary behaviours \\ School intervention
}

Childhood obesity is considered a serious public health concern in the USA. In the last 30 years, childhood obesity has tripled, and recent data suggest that one in six American children is obese and one in three is overweight or obese ${ }^{(1)}$. This dramatic increase in childhood overweight and obesity can be attributed to a number of factors, including individual child-level factors. Children's dietary behaviours and their knowledge of nutrition-related information have been identified as primary mechanisms for weight gain. For instance, consumption of foods high in saturated fat and sugary beverages is related to paediatric weight gain ${ }^{(2)}$. In contrast, fruit and vegetable intake in childhood is associated with lower risk of being overweight and obese ${ }^{(3)}$. Unfortunately, most children in the USA do not meet current dietary recommendations of at least four servings of fruits and vegetables per day $^{(4)}$, and in fact many children consume almost no servings of fruits or vegetables each day $^{(5)}$. Consumption of fruits and vegetables may be low in the USA because many children do not establish preferences for healthy foods early in life ${ }^{(6)}$. Food preferences develop in early childhood ${ }^{(6)}$ and preferences for fruits and vegetables have been positively linked with intake ${ }^{(6-8)}$ and inversely associated with risk of obesity in children $^{(7)}$. Thus, targeting preferences in young children may be a promising approach for promoting consumption of fruits and vegetables and reducing risk for overweight and obesity. Although the home environment is a critical context for establishing young children's food preferences and for promoting fruit and vegetable consumption, parents may unintentionally decrease children's preferences for fruits and vegetables by decisions concerning food availability ${ }^{(9)}$. However, repeated exposure to healthy foods, even outside the home (e.g. 
at school), can lead to improvements in children's preferences and willingness to consume these foods ${ }^{(9,10)}$.

Nutrition-related knowledge has also been identified as a mechanism for healthy weight in childhood, as this knowledge helps shape children's dietary preferences and choices $^{(11,12)}$. When children have an understanding of the benefits of including fruits and vegetables in their diets, they are more likely to choose and consume these foods ${ }^{(13)}$. Schools can serve as a primary context for the development of healthy dietary behaviours and nutritionrelated knowledge, and although evidence is mixed, previous research has found that these domains can be improved through school-based interventions ${ }^{(14-18)}$. For example, results from one study indicated that 2 nd and $3 \mathrm{rd}$ graders who participated in a nutrition education programme delivered in elementary schools demonstrated greater improvements in dietary behaviours and nutrition knowledge than children in a control group ${ }^{(17)}$; however, in that study, nutrition educators, not classroom teachers, implemented the intervention. Other studies have found that programmes focused on school-based gardens may be effective in promoting dietary behaviours and knowledge $^{(16)}$, yet many schools do not have the space, resources or expertise to implement such programmes.

Despite the importance of developing healthy dietary behaviours and nutrition and health knowledge for children's outcomes and the fact that some nutrition education programmes have been effective in elementary schools, few teachers incorporate nutrition education into their curricula $^{(19)}$. In a recent study, elementary-school teachers identified several reasons for not incorporating nutrition education into their classrooms, including a lack of suitable curriculum where lessons were feasible, easy to implement and short, and a lack of time to incorporate a stand-alone curriculum that did not align with required education standards ${ }^{(20)}$. Teachers in that study reported a preference for a curriculum that could be easily integrated into curricula from other subject areas (e.g. health, science) that included approximately ten sessions per year. Thus, the objective of the current study was to develop and evaluate a short (6-week) and feasible nutrition education curriculum that aligned with the National Health Education Standards designed to enhance children's dietary behaviours and nutrition and health knowledge. Much of the previous research exploring the utility of schoolbased interventions, such as school gardens, has utilized non-experimental or quasi-experimental designs, limiting the validity and generalizability of study findings ${ }^{(21)}$; thus in the current study we employed a rigorous randomized controlled design to evaluate the curriculum. It was hypothesized that children who participated in the curriculum would exhibit greater improvements in a direct assessment of fruit and vegetable preferences and a nutrition and health knowledge survey relative to children in a control group.

\section{Methods}

\section{Study design and procedures}

In the current study we employed a cluster randomized controlled design. Using convenience sampling methods, three elementary schools in a Midwestern US state were recruited to participate in the study during the summer of 2016. In the autumn of 2016, teachers and children from all 2nd grade classrooms in the three schools were recruited to participate in the study. Teachers were recruited through an email with an informational letter describing study procedures. Teachers then provided written consent if they were willing to participate. Children were recruited to participate in the study through an informational letter sent to their parents describing study procedures. Parents then provided written consent if they were willing to allow their child to participate. Parents of 131 children returned consent forms and were included in the study sample. Children were, on average, 8 years old (53\% female) and were primarily Caucasian (90\%), with other races/ethnicities including 1\% Asian, 3\% African American, 2\% Hispanic, 1\% mixed race and 3\% unknown. Children's parents had education levels ranging from some high school to a graduate degree, with an average level of education of a bachelor's degree.

Following parent consent procedures, all participating children completed pre-test assessments, including a short survey on nutrition and health knowledge and a direct assessment of fruit and vegetable preferences administered by their teachers. Teachers were trained to administer the assessment in a standardized fashion and were told not to add any language beyond what was provided in the assessment script to avoid the introduction of any bias. After completion of pre-test assessments, all 2nd grade classrooms ( $n$ 10) were randomly assigned within school to be intervention or business-as-usual control classrooms (school 1: one intervention, one control classroom; school 2: one intervention, one control classroom; school 3: three intervention, three control classrooms; children: eighty-two in intervention classrooms, forty-nine in control classrooms). Children in intervention classrooms were not significantly different from children in control classrooms according to age, gender, parent education, ethnicity or pre-test assessments.

Teachers in intervention classrooms participated in a $2 \mathrm{~h}$ training session conducted by project staff either at their respective schools or at the research university where the project investigators were housed. Training consisted of a review of the curriculum day-by-day, including an outline of all activities. As a support, teachers received an intervention manual with all materials required to teach the programme, including class copies of all handouts. Teachers also received a \$US 100 gift card to purchase fruits and vegetables necessary for food tastings. For consistency, questions regarding the programme or 
implementation were directed to project staff that conducted training.

Following training, teachers in intervention classrooms implemented a 6-week curriculum (two lessons per week) that was designed to promote children's nutrition knowledge and fruit and vegetable preferences. Children in the control classrooms received no intervention and teachers carried on activities as usual. To establish fidelity of implementation, intervention teachers completed a brief evaluation at the end of each week's instruction that included questions related to curriculum delivery. An example item included: 'How many lessons did you teach this week?' Items also included frequency questions, such as 'How often did you deliver the lessons as was intended?' These items were rated on a 4-point scale ( $1=$ 'rarely', $4=$ 'always'). Following intervention implementation, all participating children completed post-test assessments that were identical to pre-test assessments. All study activities were reviewed and approved by the institutional review board at a research university through an expedited review process.

\section{Intervention}

The nutrition education curriculum used in the intervention was designed to improve children's nutrition and health knowledge and preferences for fruits and vegetables. This intervention was grounded in Social Cognitive Theory ${ }^{(22)}$, which emphasizes environmental inputs that contribute to children's self-efficacy around dietary behaviours. As Bandura notes, from a social cognitive perspective, in order for school-based health promotion programmes to be effective, children must be equipped with the beliefs and skills needed to change their behaviours $^{(23)}$. Our curriculum provides children with ageappropriate background information on nutrition and health that is intended to change their belief systems around the benefits of eating healthy foods. Further, our curriculum gives children opportunities to develop the skills necessary to make healthy choices and try a variety of foods they may not have been exposed to before. Thus, our curriculum is in line with a Social Cognitive Theory approach.

The curriculum was developed during the summer of 2016 in collaboration with three 3rd grade teachers. Recruiting 3rd grade teachers was deliberate as we did not want to cut into our research pool of 2 nd grade teachers, and 3rd grade teachers begin their teaching year with 'ending' 2nd grade students. Thus, they would understand the developmental stage of 2 nd graders and be equipped to develop the curriculum. During a full-day workshop, the research team and teachers developed a 6-week curriculum, consisting of lessons implemented twice per week. The lessons were developed based on four existing programmes: MyPlate for grades 1-2 and 3-4, Two-Bite Club, and Put a Rainbow on Your Plate. As lessons were developed, they were aligned to the National Health Education Standards. This enabled teachers to cover topics required by the state while using the nutrition lessons. In addition to direct instruction on nutrition and health, a variety of age-appropriate activities were implemented throughout the programme including trivia, food tastings, projects and games.

Week 1 focused on the overarching questions, "What does it mean to be healthy?' and 'What does it mean to eat healthy?' The MyPlate framework was highlighted. As a culminating activity for day 2, students labelled a blank copy of the MyPlate diagram after learning the components of healthy meals during both lessons of week 1 . Week 2 emphasized the question, 'Why is it important to eat a variety of foods from all food groups?' The concept of nutrients was introduced and the book, Two-Bite Club, was read. The primary goal of the Two-Bite Club book is to encourage children to try at least 'two bites' of foods, and following this first reading children were challenged to try two bites of new foods as they were introduced throughout the rest of the nutrition curriculum. Week 3 focused on the questions, 'What should I eat less of and why?' and 'What can I eat instead?' This week was focused on teaching children strategies for replacing sweet or salty snacks with healthy choices. Week 3 also introduced the Put a Rainbow on Your Plate framework that was incorporated throughout the rest of the curriculum. Put a Rainbow on Your Plate encourages children to eat foods that are a variety of colours. In week 3 , children learned the nutritional benefits of red foods while sampling a variety of red fruits and vegetables. Week 4 focused on the final question to be emphasized throughout the remainder of the programme: "Why should we eat fruits and vegetables?' During this week, children learned the nutritional benefits of orange foods and sampled fruits and vegetables that are orange. The final project was also introduced to children this week. The final project served as an assessment of children's understanding of key concepts introduced throughout the curriculum. The curriculum provided each class a choice from one of three projects: (i) children creating 'Eat the Rainbow' posters to be placed in the school cafeteria; (ii) children developing video commercials for various fruits and vegetables to be played on the school news; or (iii) children holding a farmers' market (with pretend or real foods) to 'sell' to students in other grades or to school adults. Each project required children to report on the nutritional benefits of fruits and vegetables. Weeks 5 and 6 focused on the continuing theme of 'Why should we eat fruits and vegetables?' and emphasized the nutritional value and tasting of green and blue/ purple foods, respectively. The final projects were presented in week 6.

Overall, intervention implementation fidelity was high. On average, over the course of 6 weeks, teachers reported delivering two lessons per week (mean 2.00 (SD 0.11)) that lasted approximately $15-20 \mathrm{~min}$ each, which was the 
targeted duration and dosage. Teachers also indicated that, on average, they implemented the lessons as intended (mean 3.65 (SD 0.42); 4-point scale). Finally, teachers reported that children were engaged in and enjoyed the lessons (mean 3.56 (sD 0.46) and mean 3.57 (SD 0.58), respectively; 4-point scale).

\section{Measurement instruments}

Parents completed a demographic survey including items related to children's age, gender and ethnicity, as well as their own education level. Two child-level assessments were used in the present study to test the efficacy of the intervention. The first assessment was developed by the research team to align with nutrition and health content provided in the intervention. This survey consisted of fifteen questions. Sample items included: "What contains the most vitamin A per serving?' (response options: $1=$ 'oranges', $2=$ 'cantaloupe') and 'What you eat can make a difference in your chances of getting heart diseases or cancer' (response options: $1=$ 'true', 2 ='false'). Children received a score of 1 for each correct response and 0 for each incorrect response, with a possible range in scores from 0 to 15 . The internal consistency (Cronbach's $\alpha$ ) for this outcome measure was 0.79 .

The second assessment was a direct measure of children's fruit and vegetable preferences that was modified from an assessment used in previous research ${ }^{(2)}$. Teachers administered this measure to participating children. During this assessment, teachers showed children forty-one coloured pictures of a variety of fruits and vegetables. While teachers were showing children a picture of a particular fruit or vegetable, they asked 'What is your opinion of this [food]?' Children were asked to respond with 'I like it' (score of 2), 'I don't like it' (score of 1) or 'I've never tried it' (score of 0 ). Scores across all fruits and vegetables were summed, with a total range in scores from 0 to 82 . The internal consistency for this outcome measure was $0 \cdot 90$.

\section{Analytic procedures}

Data analyses were conducted using the statistical software package Stata version 13.0. All outcome data were analysed for normality using skewness and kurtosis checks. Preliminary analyses, including $t$ tests, were conducted to explore differences in outcome scores between the intervention and control children. Data were multilevel, with randomization occurring at the classroom level and child-level data being the unit of analysis. Intraclass correlations representing classroom variance in outcomes were 0.51 for the nutrition and health knowledge survey and 0.02 for the food preferences assessment. To account for this variance, multiple regression analyses that used the generalized Huber-White sandwich estimator to adjust SE errors for non-independence (clustering by classroom) were conducted. Separate models were run for each outcome. Models included the following covariates: pre-test scores, age, gender, ethnicity and parent education. Statistical significance was determined using an $\alpha$ level of $0 \cdot 05$. There were very few missing data $(<1 \%$ for nutrition and health knowledge, $<2 \%$ for food preferences); however, full information maximum likelihood was employed to handle missing data.

Table 1 Descriptive characteristics of the full sample and intervention and control groups of elementary-school children participating in the nutrition education curriculum intervention in a Midwestern US state, autumn 2016

\begin{tabular}{|c|c|c|c|c|c|c|c|}
\hline & \multicolumn{2}{|c|}{ Full sample $(n 131)$} & \multicolumn{2}{|c|}{ Intervention group ( $n$ 82) } & \multicolumn{2}{|c|}{ Control group (n 49) } & \multirow[b]{2}{*}{$t$} \\
\hline & Mean or \% & SD & Mean or \% & SD & Mean or \% & SD & \\
\hline Age (months) & 94.56 & 8.64 & 95.04 & $10 \cdot 35$ & 93.79 & 4.62 & $t_{(125)}=-0.79$ \\
\hline Parent education $\ddagger$ & 4.64 & 0.91 & 4.65 & 0.85 & 4.63 & 1.02 & $t_{(125)}=-0.12$ \\
\hline T1 Food pref & $39 \cdot 38$ & $13 \cdot 21$ & 38.96 & $12 \cdot 34$ & 40.06 & 14.61 & $t_{(126)}=0.46$ \\
\hline T2 Food pref & $45 \cdot 90$ & 13.88 & 47.57 & $12 \cdot 71$ & 43.08 & $15 \cdot 39$ & $t_{(127)}=-1.79^{(*)}$ \\
\hline T1 Health & 6.53 & 1.89 & $6 \cdot 71$ & $2 \cdot 15$ & $6 \cdot 24$ & 1.33 & $t_{(126)}=-1.36$ \\
\hline T2 Health & $9 \cdot 35$ & $3 \cdot 33$ & $10 \cdot 60$ & 3.04 & $7 \cdot 21$ & 2.66 & $t_{(128)}=-6 \cdot 42^{\star * *}$ \\
\hline Gender (\%) & & & & & & & $t_{(127)}=0.29$ \\
\hline Male & $46 \cdot 51$ & - & $46 \cdot 34$ & - & 44.90 & - & - \\
\hline Female & 53.49 & - & $51 \cdot 22$ & - & $55 \cdot 10$ & - & - \\
\hline Race & & & & & & & $t_{(125)}=-1.28$ \\
\hline Asian/Pacific Islander & 1.57 & - & 0.00 & - & $4 \cdot 26$ & - & - \\
\hline Back/African American & $2 \cdot 37$ & - & $2 \cdot 50$ & - & $2 \cdot 13$ & - & - \\
\hline Hispanic American & 1.57 & - & 1.25 & - & 2.13 & - & - \\
\hline White/Caucasian & 92.92 & - & 95.0 & - & 89.36 & - & - \\
\hline Multiracial & 1.57 & - & $1 \cdot 25$ & - & $2 \cdot 13$ & - & - \\
\hline
\end{tabular}

T1, pre-test assessment; Food pref, food preferences; T2, post-test assessment; Health, nutrition and health knowledge.

(*) $P<0.10,{ }^{* \star *} P<0.001$.

$\dagger t$ Tests comparing intervention and control groups.

$\ddagger 1=8$ th grade or less, $2=$ some high school, $3=$ General Educational Development, $4=$ high school diploma, $5=$ some college, 6 = associate's degree, 7 = bachelor's degree, $8=$ master's degree, $9=$ doctoral $/$ postgraduate degree. 


\section{Results}

Descriptive statistics and correlations are presented in Tables 1 and 2. Intervention participation was marginally correlated with food preferences at post-test and significantly related to the nutrition and health survey. Skewness and kurtosis analyses indicated normal distributions for both outcomes. Results from analyses revealed that when controlling for the pre-test nutrition and health survey score, age, gender, ethnicity and parent education, children in the intervention condition demonstrated significantly higher scores on the nutrition and health survey compared with children in the control condition $(\beta=0.47, P=0 \cdot 001)$. Similarly, results suggested that when controlling for the pre-test fruit and vegetable preferences, age, gender, ethnicity and parent education, children who received the intervention showed greater preferences for fruits and vegetables at post-test than children who did not receive the intervention $(\beta=0 \cdot 19$, $P=0 \cdot 003)$. See Table 3 for a summary of results and effect sizes.

\section{Discussion}

The aim of the current study was to develop and evaluate a feasible nutrition education curriculum designed to enhance children's dietary behaviours and nutrition and health knowledge. As expected, results from a randomized controlled trial indicated that 2nd grade children who participated in the curriculum demonstrated greater improvements in their fruit and vegetable preferences and nutrition and health knowledge relative to children in a control group. These findings suggest that a relatively short, 6-week intervention that aligns with educational standards has the ability to significantly enhance children's outcomes and thus may be a more feasible option for teachers to incorporate into their classrooms than what is currently available.

Childhood obesity, and related health problems, has become a significant public health issue in the USA in recent years ${ }^{(1)}$. In the current study, we chose to focus on designing an intervention that targeted children's dietary behaviours (i.e. food preferences) and nutrition-related knowledge because of evidence suggesting that both have can a substantial impact on sustained behavioural change regarding consumption of healthy foods ${ }^{(7,11,12)}$. For example, in a review, Blanchette and Brug noted that taste preferences are the among most critical determinants of children's consumption of fruits and vegetables. Knowledge of recommended fruit and vegetable intakes was also identified as a key determinant of behaviour change ${ }^{(24)}$. As such, we developed a short and feasible nutrition education curriculum that aligns with the National Health Education Standards. Social Cognitive Theory was used as a basis for our intervention design; the intent was to equip children with the necessary skills and beliefs to effect actual behavioural changes ${ }^{(23)}$. For example, by providing children with nutrition and health knowledge in a fun and engaging manner, the goal was to facilitate a sense of selfefficacy for making healthy choices. Our findings indicated that just two, 15-20 min lessons per week for 6 weeks were enjoyable for children and feasible for teachers. Further, participation in these lessons effected positive change in both targeted outcomes (food preferences and nutrition and health knowledge).

The findings with regard to nutrition education are in line with other school-based and nutrition-focused interventions $^{(14-18)}$. For instance, in one study of similar duration, 5th grade children who participated in a nutrition education programme at school showed significantly greater gains in nutrition knowledge relative to a control group $^{(14)}$. Similarly, in another study, 2nd and 3rd grade children exposed to six weekly nutrition education lessons demonstrated greater improvements in nutrition knowledge compared with children in a control condition ${ }^{(17)}$. There have been few school-based and nutrition-focused interventions that have been effective for promoting children's fruit and vegetable preferences. For example, in a multicomponent, school-based intervention study, although there were intervention effects on nutrition knowledge and vegetable identification, there were no

Table 2 Correlation matrix for all study variables $(n$ 131)

\begin{tabular}{|c|c|c|c|c|c|c|c|c|c|}
\hline Variable & 1 & 2 & 3 & 4 & 5 & 6 & 7 & 8 & 9 \\
\hline 1. Age† & - & & & & & & & & \\
\hline 2. Gender & -0.02 & - & & & & & & & \\
\hline 3. Ethnicity & -0.01 & -0.01 & - & & & & & & \\
\hline 4. Parent education $\ddagger$ & -0.05 & 0.00 & $0.21^{*}$ & - & & & & & \\
\hline 5. Condition & 0.07 & -0.03 & 0.11 & 0.01 & - & & & & \\
\hline 6. T1 Food pref & -0.08 & -0.13 & -0.09 & $-0.24^{\star *}$ & 0.04 & - & & & \\
\hline 7. T2 Food pref & -0.01 & -0.06 & -0.13 & -0.09 & $0 \cdot 16^{(*)}$ & $0.49^{\star \star \star}$ & - & & \\
\hline 8. T1 Health & 0.08 & -0.07 & 0.07 & -0.01 & 0.12 & $0 \cdot 19^{\star}$ & 0.04 & - & \\
\hline 9. T2 Health & 0.04 & 0.04 & $0 \cdot 16^{(*)}$ & 0.08 & $0.49^{\star \star \star}$ & -0.01 & $0.19^{*}$ & $0.22^{*}$ & - \\
\hline
\end{tabular}

T1, pre-test assessment; Food pref, food preferences; T2, post-test assessment; Health, nutrition and health knowledge.

${ }_{(*)} P<0.10,{ }^{\star} P<0.05,{ }^{* *} P<0.01,{ }^{* * *} P<0.001$.

tChild age measured in months.

$\ddagger 1=8$ th grade or less, $2=$ some high school, $3=$ General Educational Development, $4=$ high school diploma, $5=$ some college, $6=$ associate's degree,

$7=$ bachelor's degree, $8=$ master's degree, $9=$ doctoral/postgraduate degree. 
Table 3 Effects of the nutrition education curriculum intervention in a Midwestern US state on elementary-school children's food preferences and nutrition and health knowledge, autumn 2016

\begin{tabular}{|c|c|c|c|c|c|c|c|c|}
\hline & \multicolumn{4}{|c|}{ Food preferences } & \multicolumn{4}{|c|}{ Nutrition and health knowledge } \\
\hline & $B$ & SE & $\beta$ & Hedge's $g$ & $B$ & SE & $\beta$ & Hedge's $g$ \\
\hline Age & 0.03 & 0.04 & 0.02 & & -0.00 & 0.03 & -0.00 & \\
\hline Parent education & 0.65 & 0.65 & 0.04 & & 0.18 & $0 \cdot 19$ & 0.05 & \\
\hline Gender & 0.34 & 1.35 & 0.01 & & 0.42 & 0.35 & 0.06 & \\
\hline Ethnicity & $-3 \cdot 16^{\star}$ & 1.27 & -0.12 & & 0.57 & 0.71 & 0.09 & \\
\hline Pre-test score & $0.52^{\star \star \star}$ & 0.10 & 0.50 & & $0.29^{*}$ & 0.12 & 0.16 & \\
\hline Condition & $5 \cdot 28^{\star \star}$ & 1.54 & $0 \cdot 19$ & 0.39 & $3 \cdot 19^{\star \star}$ & $1 \cdot 21$ & 0.47 & 1.11 \\
\hline
\end{tabular}

${ }^{*} P<0.05,{ }^{* *} P<0.01,{ }^{\star \star \star} P<0.001$.

significant effects on children's food preferences ${ }^{(18)}$. The majority of school-based interventions that have been effective in improving food preferences include a garden component. Morris and Zidenberg-Cherr found that although a nutrition education curriculum did produce favourable effects on food preferences for 4th grade children, effects were greater and sustained when garden activities were included ${ }^{(25)}$. Although garden-based interventions have promise for improving food preferences, they may not be feasible for many schools. Gardens can be expensive to build and time-consuming to maintain, and some schools may not have the resources or space for this endeavour. Thus, one goal of the current study was to include other activities that may promote nutrition knowledge and food preferences that did not include a garden component.

One key ingredient of our curriculum that may have facilitated positive effects on both dietary behaviours and nutrition and health knowledge is the inclusion of tastings. For young children, nutrition education may not always be enough to effect actual change in children's dietary behaviours; however, exposure to healthy fruits and vegetables with tastings has been shown to promote preferences for these foods ${ }^{(26)}$. As part of our nutrition education curriculum, children were exposed to and tasted a variety of fruits and vegetables that were introduced in fun and engaging ways. For example, in week 2 , children were introduced to the Two-Bite Club via a storybook reading that encourages children to try at least two bites of new foods, just like the characters in the Two-Bite Club book. Then, children were given new foods to taste (e.g. pomegranate, butternut squash, okra). This inclusion of tastings along with nutrition education may be critical for promoting healthy dietary behaviours in young children. It will be important for future research to test this hypothesis.

An additional strength of the present study is the potential for the curriculum to be integrated into other content domains. Although the curriculum primarily targets nutritional components of the National Health Education Standards, it could also meet some of the National Health Science Standards. For example, one of the foci of the 'Science in Personal and Social Perspectives' standard is personal health. Teachers could use the curriculum to meet this particular standard in addition to meeting health education standards.

Although the current study has a number of strengths (e.g. randomized controlled design), limitations must be noted. First, our sample size was relatively small and homogeneous. It will be critical for future research to replicate our findings with larger and more diverse samples. Second, our targeted outcomes were limited to direct assessment of food preferences and child report of nutrition and health knowledge. Although both of these outcomes have been associated with consumption in previous research ${ }^{(1,11,12)}$, future research evaluating the curriculum would benefit from the inclusion of pre- and post-test assessments of fruit and vegetable consumption. Finally, although the short-term intervention was found to effective, we did not have a long-term follow-up to assess the extent to which the effects were maintained. It is possible that the intensity of the intervention was not enough to lead to sustained behaviour change. Future research should test whether a more holistic approach to curriculum that more fully integrates nutrition into all areas of education (e.g. health, maths, science, arts) would lead to even stronger effects in the short and long term.

Despite these limitations, our study supports the inclusion and effectiveness of a nutrition education programme for promoting young children's fruit and vegetable preferences and nutrition and health knowledge. Preferences for and knowledge of fruits and vegetables predict children's consumption of healthy foods ${ }^{(1,11)}$. Thus, exposing children to healthy foods and providing an education on the value of these foods early in life may help prevent the onset of overweight and obesity, laying the foundation for lifelong health and well-being.

\section{Acknowledgements}

Financial support: This study was supported by a grant from the Indiana State Department of Agriculture, Specialty Crop Block Grant Program (EDS \#A337-15-SCBG-14006). The funder had no role in the design, analysis or writing of this article. Conflict of interest: None. Authorship: S.A.S. designed the study, recruited participants, collected data, trained teachers, analysed the data and led 
manuscript preparation. L.M.B. managed data, analysed the data and assisted in manuscript preparation. I.K. managed data, analysed the data and assisted in manuscript preparation. L.K. recruited participants, collected data, trained teachers and assisted in manuscript preparation. B.K. helped design the study, collected data and assisted in manuscript preparation. T.B. managed the grant and assisted in manuscript preparation. Ethics of human subject participation: This study was conducted according to the guidelines laid down in the Declaration of Helsinki and all procedures involving human subjects were approved by the Purdue University Institutional Review Board. Written (from parents) and verbal (from children) informed consent was obtained from all subjects. Verbal consent was witnessed and formally recorded.

\section{References}

1. Ogden CL, Carroll MD \& Lawman HG (2016) Trends in obesity prevalence among chidlren and adolescents in the United States, 1988-1994 through 2013-2014. JAMA 315, 2292-2299.

2. Gillis LJ, Kennedy LC, Gillis AM et al. (2002) Relationship between juvenile obesity, dietary energy and fat intake and physical activity. Int J Obes Relat Metab Disord 26, 458-463.

3. Lakkakula AP, Zanovec M, Silverman L et al. (2008) Black children with high preferences for fruits and vegetables are at less risk of being at risk of overweight or overweight. $J$ Am Diet Assoc 108, 1912-1915.

4. US Department of Agriculture \& US Department of Health and Human Services (2010) Dietary Guidelines for Americans 2010, 7th ed. Washington, DC: US Government Printing Office.

5. Siega-Riz AM, Deming DM, Reidy KC et al. (2010) Food consumption patterns of infants and toddlers: where are we now? J Am Diet Assoc 110, 12 Suppl., S38-S51.

6. Birch LL (1998) Development of food acceptance patterns in the first years of life. Proc Nutr Soc 57, 617-624.

7. Domel S, Thompson W, Davis H et al. (1996) Psychosocial predictors of fruit and vegetable consumption among elementary school children. Health Educ Res 11, 299-308.

8. Domel S \& Thompson W (2002) Fourth-grade children's consumption of fruit and vegetable items available as part of school lunches is closely related to preferences. J Nutr Educ Behav 34, 166-171.

9. Benton D (2004) Role of parents in the determination of the food preferences of children and the development of obesity. Int J Obes Relat Metab Disord 28, 858-869.

10. Anzman-Frasca S, Savage JS, Marini ME et al. (2012) Repeated exposure and associative conditioning promote preschool children's liking of vegetables. Appetite 58, 543-553.
11. Contento I, Balch FI \& Bronner YL (1995) Nutrition education for school-aged children. J Nutr Educ 27, 298-311.

12. Asakura K, Todoriki H \& Sasaki S (2017) Relationships between nutrition knowledge and dietary intake among primary school children in Japan: combined effect of children's and their guardian's knowledge. J Epidemiol 27, 483-491.

13. Perez-Rodrigo C \& Aranceta J (2001) School-based nutrition education: lessons learned and new perspectives. Public Health Nutr 4, 131-139.

14. Kandiah J \& Jones C (2002) Nutrition knowledge and food choices of elementary school children. Early Child Dev Care 172, 269-273.

15. Panunzio MF, Antonicello A, Pisano A et al. (2007) Nutrition education intervention by teachers may promote fruit and vegetable consumption in Italian students. Nutr Res 27, 524-528.

16. Parmer SM, Salisbury-Glennon J, Shannon D et al. (2009) School gardens: an experiential learning approach for a nutrition education program to increase fruit and vegetable knowledge, preference, and consumption among second grade students. J Nutr Educ Behav 41, 212-217.

17. Powers AR, Struempler BJ, Guarino A et al. (2005) Effects of a nutrition education program on the dietary behavior and nutrition knowledge of second-grade and third-grade students. J Sch Health 75, 129-133.

18. Scherr RE, Linnell JD, Dharmar M et al. (2017) A multicomponent, school-based intervention, the Shaping Healthy Choices Program, improves nutrition-related outcomes. $J$ Nutr Educ Behav 49, 368-379.

19. Kann L, Telljohann SK \& Wooley SF (2007) Health education: results from the School Health Policies and Programs Study. J Sch Health 77, 408-434.

20. Perera T, Frei S, Frei B et al. (2015) Improving nutrition education in US elementary schools: xhallenges and opportunities. J Educ Pract 6, 41-50.

21. DeCosta P, Møller P, Bom-Frost M et al. (2017) Changing children's eating behavior: a review of experimental research. Appetite 113, 327-357.

22. Bandura A (1986) Social Foundations of Thought and Action: A Social Cognitive Theory. Englewood Cliffs, NJ: Prentice Hall.

23. Bandura A (2004) Health promotion by social cognitive means. Health Educ Behav 31, 143-164.

24. Blanchette L \& Brug J (2005) Determinants of fruit and vegetable consumption among 6-12-year-old children and effective interventions to increase consumption. J Hum Nutr Diet 18, 431-443.

25. Morris JL \& Zidenberg-Cherr S (2002) Garden-enhanced nutrition curriculum improves fourth-grade school children's knowledge of nutrition and preferences for some vegetables. J Am Diet Assoc 102, 91-93.

26. Wardle J, Herrera ML, Cooke L et al. (2003) Modifying children's food preferences: the effects of exposure and reward on acceptance of an unfamiliar vegetable. Eur J Clin Nutr 57, 341-348. 